\title{
Modification of PMMA Bone Cement for Enhancing Applicability in Vertebroplasty
}

\author{
Ching-Lung Tai ${ }^{1 *}$, Wei-De Lin², Yen-Chen Lee ${ }^{1}$, Mu-Yi Liu', Lih-Huei Chen ${ }^{2}$, Po-Liang Lai ${ }^{2}$ \\ ${ }^{1}$ Graduate Institute of Medical Mechatronics/Chang Gung University \\ 259, Wen-Hua 1st RD., Kweishan, Taoyuan, Taiwan \\ taicl@mail.cgu.edu.tw; asdfmark200@hotmail.com; zero_790723@hotmail.com \\ ${ }^{2}$ Department of Orthopedic Surgery/Chang Gung Memorial Hospital \\ 5, Fu-Hsin St., Kweishan, Taoyuan, Taiwan \\ linwate@gmail.com; lhchen2132@cgmh.org.tw; polianglai@gmail.com
}

\section{Extended Abstract}

Polymethylmethacrylate (PMMA) bone cement has been widely used as bone void filler for vertebroplasty. However, the application of PMMA in vertebroplasty has some drawbacks. These include the material's short handling time, exothermic polymerization, and excessive stiffness [1,2]. Various techniques have been reported to enhance the applicability of PMMA in vertebroplasty. Previous literatures had demonstrated that the addition of castor oil to PMMA bone cement could change its mechanical properties by lowering its Young's modulus, compressive strength, and reacting temperature [3,4]. Additionally, our previous study indicated that pre-cooling PMMA material effectively slowed its polymerization reaction and thus lengthened its handling time in vertebroplasty [5]. Pre-cooling and the addition of castor oil are simple and inexpensive methods to enhance the applicability of PMMA for clinical settings. However, how PMMA changes after such treatments remains unknown. This study aims to create an ideal modified PMMA cement to solve the above-mentioned problems.

Modified PMMA cements were prepared by combining PMMA with three different volume fractions of castor oil (5\%, $10 \%$ and $15 \%$ ). The peak polymerization temperatures, times to achieve the peak polymerization temperature, porosities, densities, modulus and maximum compression strengths of standard (without castor oil) and modified cements were investigated following storage at ambient temperature $\left(22^{\circ} \mathrm{C}\right)$ or under pre-cooling conditions $\left(3^{\circ} \mathrm{C}\right)$.

Our results revealed that increasing castor oil content and pre-cooling treatment effectively decreased the peak polymerization temperatures and increased the duration to achieve the peak polymerization temperature $(p<0.05)$. Furthermore, the mechanical properties of the modified PMMA cement, including density, modulus and maximum compression strength, decreased with increasing castor oil content. However, preparation temperature (room temperature vs. pre-cooling) had no significant effect $(\mathrm{p}>0.05)$ on these mechanical properties.

In conclusion, the addition of castor oil to PMMA followed by pre-cooling created an ideal modified bone cement with a low modulus, low polymerization temperature and long handling time, enhancing its applicability and safety for vertebroplasty.

\section{References}

[1] Y. J. Rho, W. J. Choe, and Y. I. Chun, "Risk factors predicting the new symptomatic vertebral compression fractures after percutaneous vertebroplasty or kyphoplasty," Eur. Spine J., vol. 21, no. 5, pp. 905-911, 2012.

[2] S. M. Belkoff and S. Molloy, "Temperature measurement during polymerization of polymethylmethacrylate cement used for vertebroplasty," Spine, vol. 28, no. 14, pp. 1555-1559, 2003.

[3] E. Unosson, "Mechanical properties and spreading characteristics of bone cement for spinal applications," Luleå University of Technology: Luleå, Sweden, 2010.

[4] A. López, A. Hoess, T. Thersleff, M. Ott, H. Engqvist, and C. Persson, "Low-modulus PMMA bone cement modified with castor oil," Biomed. Mater. Eng., vol. 21, pp. 323-332, 2011.

[5] P. L. Lai, C. L. Tai, I. M. Chu, T. S. Fu, L. H. Chen, and W. J. Chen, "Hypothermic manipulation of bone cement can extend the handling time during vertebroplasty," BMC Musculoskelet. Disord., vol. 13, pp. 198, 2012. 